\title{
Survival Impact of Adjuvant Therapy in Salivary Gland Cancers following Resection and Neck Dissection
}

\author{
Aro, Katri
}

2019-06

Aro , K, Ho , A S , Luu , M , Kim , S , Tighiouart , M , Yoshida, E J , Mallen-St Clair , J ,

Shiao , S L , Leivo , I \& Zumsteg , Z S 2019 , ' Survival Impact of Adjuvant Therapy in

Salivary Gland Cancers following Resection and Neck Dissection ' , Otolaryngology - Head

and Neck Surgery , vol. 160 , no. 6 , pp. 1048-1057 . https://doi.org/10.1177/0194599819827851

http://hdl.handle.net/10138/318791

https://doi.org/10.1177/0194599819827851

publishedVersion

Downloaded from Helda, University of Helsinki institutional repository.

This is an electronic reprint of the original article.

This reprint may differ from the original in pagination and typographic detail.

Please cite the original version. 


\section{Survival Impact of Adjuvant Therapy in Salivary Gland Cancers following Resection and Neck Dissection}

\author{
Katri Aro, MD, PhD ${ }^{1,2}$, Allen S. Ho, MD ${ }^{2,3}$, Michael Luu, MPH ${ }^{4}$, \\ Sungjin Kim, $\mathbf{M S}^{4}$, Mourad Tighiouart, $\mathrm{PhD}^{4}$, Emi J. Yoshida, $\mathbf{M D}^{2,5}$, \\ Jon Mallen-St Clair, MD ${ }^{2,3}$, Stephen L. Shiao, MD, $\mathbf{P h D}^{2,5}$, \\ IImo Leivo, MD, PhD ${ }^{6}$, and Zachary S. Zumsteg, MD ${ }^{2,5}$
}

Otolaryngology-

Head and Neck Surgery

2019, Vol. 160(6) 1048-1057

(C) American Academy of

Otolaryngology-Head and Neck

Surgery Foundation 2019

Reprints and permission:

sagepub.com/journalsPermissions.nav DOI: $10.1177 / 019459981982785$ | http://otojournal.org

@SAGE
Sponsorships or competing interests that may be relevant to content are disclosed at the end of this article.

\begin{abstract}
Objective. To evaluate the impact of postoperative radiotherapy (PORT) and chemotherapy on survival in salivary gland cancer (SGC) treated with curative-intent local resection and neck dissection.

Study Design. Retrospective population-based cohort study.

Setting. National Cancer Database.

Subjects and Methods. Patients with SGC who were undergoing surgery were identified from the National Cancer Database between 2004 and 2013. Neck dissection removing a minimum of 10 lymph nodes was required. Because PORT violated the proportional hazards assumption, this variable was treated as a time-dependent covariate.
\end{abstract}

Results. Overall, 4I45 cases met inclusion criteria (median follow-up, 54 months). PORT was associated with improved overall survival in multivariable analysis, both $\leq 9$ months from diagnosis (hazard ratio [HR], 0.26; $95 \% \mathrm{Cl}, 0.20-0.34$; $P<.00 \mathrm{I})$ and $>9$ months (HR, 0.75; $95 \% \mathrm{Cl}, 0.66-0.86 ; P<$ $.00 \mathrm{I})$. In propensity score-matched cohorts, 5 -year overall survival was $67.1 \%$ and $60.6 \%$ with PORT and observation, respectively $(P<.00 \mathrm{I})$. Similar results were observed in landmark analysis of patients surviving at least 6 months following diagnosis. Adjuvant chemotherapy was not associated with improved survival (HR, I. I5; 95\% Cl, 0.99-1.34; $P=.06$ ).

Conclusion. PORT, but not chemotherapy, is associated with improved survival among patients with SGC for whom neck dissection was deemed necessary. These results are not applicable to low-risk SGCs not requiring neck dissection.

\section{Keywords}

salivary gland cancer, metastasis, radiotherapy, survival

Received September 22, 20I8; revised December 12, 2018; accepted January II, 2019.
$O$ alivary gland cancer (SGC) is a rare malignancy accounting for approximately $3 \%$ to $6 \%$ of all head and neck cancers. ${ }^{1}$ SGC can arise from the major and minor salivary glands throughout the head and neck, leading to diverse clinical presentations. Biologically, SGC is highly heterogeneous, with 20 histological subtypes recognized by the 2017 World Health Organization classification system. ${ }^{2}$ For these reasons, the diagnosis and treatment of SGC can be challenging, even at high-volume tertiary referral centers.

The cornerstone of treatment for SGC is surgical resection, but there are limited high-level data available to guide adjuvant treatment recommendations. Based on retrospective studies, radiotherapy (RT) in the curative setting is generally recommended postoperatively for T3-T4 tumors, high-grade histology, perineural or lymphovascular invasion, close or positive surgical resection margins, and multiple positive cervical lymph nodes (LNs), which are known high-risk features for increased recurrence. ${ }^{3-6}$ Although RT is widely accepted to improve locoregional control for aggressive salivary cancers, it likely does not reduce the risk of distant metastases, ${ }^{7}$ and its effect on survival is not well characterized. Several previous analyses attempted to address this with large databases, ${ }^{8-10}$ but these analyses had varying degrees of limitations - including nonstandardized surgical management of the regional LNs; not adjusting for

\footnotetext{
'Department of Otorhinolaryngology-Head and Neck Surgery, University of Helsinki and Helsinki University Hospital, Helsinki, Finland

${ }^{2}$ Samuel Oschin Comprehensive Cancer Institute, Cedars-Sinai Medical Center, Los Angeles, California, USA

${ }^{3}$ Department of Surgery, Cedars-Sinai Medical Center, Los Angeles, California, USA

${ }^{4}$ Department of Biostatistics and Bioinformatics, Cedars-Sinai Medical Center, Los Angeles, California, USA

${ }^{5}$ Department of Radiation Oncology, Cedars-Sinai Medical Center, Los Angeles, California, USA

${ }^{6}$ Institute of Biomedicine, Department of Pathology, University of Turku and Turku University Hospital, Turku, Finland
}

Corresponding Author:

Zachary S. Zumsteg, MD, Department of Radiation Oncology, Cedars-Sinai Medical Center, 8700 Beverly Blvd, Los Angeles, CA 90048, USA.

Email: zachary.zumsteg@cshs.org 
immortal time bias, given that patients dying immediately after surgery are unable to receive adjuvant therapy; inclusion of numerous unknown covariables without imputation; and the lack of a validity assessment for the Cox proportional hazards assumptions.

In the present study, we explored the impact of postoperative RT (PORT) with and without chemotherapy on survival among patients with localized SGC who were undergoing curative-intent resection and neck dissection per the National Cancer Database (NCDB), using various statistical techniques to mitigate the limitations of previous analyses. Moreover, we performed subgroup analyses to try to identify factors associated with a greatest benefit from PORT.

\section{Material and Methods Data Source}

The NCDB is a hospital-based registry representing approximately $70 \%$ of all newly diagnosed cancer cases in the United States. It comprises data from $>1500$ commissionaccredited cancer programs. ${ }^{11}$ The NCDB is a registry maintained by the Commission on Cancer of the American College of Surgeons and the American Cancer Society. There are established criteria to certify the quality of the submitted data, as well as an application process to obtain the data. However, upon distribution of the data, the Commission on Cancer of the American College of Surgeons and the American Cancer Society are not responsible for the analysis and conclusions presented in this article. All data in this study were abstracted from the NCDB, de-identified, and investigated, covering patients diagnosed from 2004 to 2013. This study was deemed exempt by the Cedars-Sinai Medical Center institutional review board.

\section{Patient Selection}

Our study included patients with nonmetastatic carcinoma of the head and neck with salivary gland-specific histology undergoing curative-intent surgical resection. We excluded all squamous cell carcinomas even if they involved the major salivary glands, given that these commonly represent LN metastases from cutaneous head and neck sites. We also required that all patients undergo neck dissection to minimize bias related to pathologic nodal staging assessment and to allow accurate matching and stage adjustment in multivariable models. We defined neck dissection as a procedure yielding at least $10 \mathrm{LNs}$ for pathologic assessment to exclude more limited biopsies or incidentally detected LNs in the primary specimen.

We queried the NCDB for head and neck cancers diagnosed between 2004 and 2013 with salivary histologies ( $\mathrm{n}=34,959)$ according to the World Health Organization's classification. $^{1,2}$ The CONSORT diagram (Consolidated Standards of Reporting Trials) describes the patients included in this analysis (see Supplemental Figure S1, available in the online version of the article). Patients with the following characteristics were excluded: noninvasive histology $(n=63)$, distant metastasis at presentation $(n=1850)$, unknown follow-up details $(\mathrm{n}=3536)$, no surgery at the primary site $(\mathrm{n}=186)$, unknown pathologic $\mathrm{T}$ classification $(\mathrm{n}=397)$, no documented LN staging $(\mathrm{n}=12,364)$, unknown number of LNs examined $(\mathrm{n}=420),<10$ LNs examined $(\mathrm{n}=10,354)$, RT received prior to surgery or unknown timing of RT $(\mathrm{n}=311)$, unknown RT dose $(\mathrm{n}=$ 326), and unknown details regarding the receipt of chemotherapy $(n=795)$ or the timing of chemotherapy with respect to RT $(n=73)$. We also excluded patients with adenosquamous histology $(\mathrm{n}=139)$, as this is regarded as a malignancy of the surface epithelium and not the salivary glands. ${ }^{2}$ This left 4145 patients, who formed the study cohort. Multiple imputation was performed to account for missing values of covariates. However, a confirmatory subgroup analysis was performed for patients who had complete data for significant predictors included in the model. Patients were defined as receiving PORT if they received at least 50 Gy. However, a sensitivity analysis was also performed defining PORT as any radiation regardless of dose. Concomitant chemoradiation (CRT) was defined as the initiation of chemotherapy between the 14 days before the start of RT and the 14 days after. The $20 \%$ of patients treated at the highest-volume facilities were defined as receiving treatment at high-volume facilities; all other patients were considered to have received treatment at lower-volume facilities.

\section{Statistical Analysis}

Missing data patterns for variables with missing values were assessed and examined with the method proposed by Little. ${ }^{12}$ Missing-ness rates were $26.5 \%$ for grade, $25.0 \%$ for extranodal extension (ENE), and $4.7 \%$ for margins and were found to be not missing completely at random. To reduce the potential for bias, missing values were imputed by a multiple imputation technique with fully conditional specifications implemented by the MICE algorithm described by Van Buuren and Groothuis-Oudshoorn. ${ }^{13,14}$

The primary purpose of this study was to determine the association of PORT with overall survival (OS) in SGC. Baseline characteristics of patients undergoing surgery alone versus PORT or CRT were compared with 2-sample $t$ tests for age and with chi-square tests for categorical covariates. The median follow-up time was calculated with the reverse Kaplan-Meier method. The primary outcome was OS calculated from the date of diagnosis to the date of death or censored at last follow-up. Estimated survival functions were generated via the Kaplan-Meier method and compared with a log-rank test.

Univariate and multivariable survival analyses were performed with the Cox proportional hazards model. Variable selection was performed with a backward stepwise method, with optimization for Akaike information criterion. ${ }^{15}$ Because the main covariate of interest, PORT, violated the proportional hazards assumption as assessed by Schoenfeld residuals, PORT was modeled as a time-varying coefficient. ${ }^{16}$ The extended Cox model was used in conjunction with the Heaviside step function, where the extended Cox model yields constant hazard ratios (HRs) for PORT among different time intervals. ${ }^{17}$ We accomplished this by 
dichotomizing our data set into time-dependent epochs at 9 months and analyzing in a "counting process" format. The cut point at 9 months was chosen because this cut point resulted in the lowest Akaike information criterion with both time segments satisfying the proportional hazards assumption. The effect of PORT was then estimated with the extended Cox model at $\leq 9$ and $>9$ months from diagnosis. Multicollinearity among covariates was assessed by the variable inflation factor and deemed nonproblematic.

Because of the possibility of immortal time bias in a retrospective study of adjuvant treatment (ie, patients need to survive a certain amount of time after surgery to receive PORT), we also performed landmark analyses including only patients surviving $\geq 6$ months to exclude those who died prior to being able to complete PORT.

Propensity scores were calculated by fitting a multivariable logistic regression model, adjusting for covariates (see Supplemental Table S1, available in the online version of the article). Due to the imbalances of our treatment and control groups, caliper matching with a caliper of 0.01 was used in conjunction with the nearest-neighbor algorithm to improve the overall match result. ${ }^{18}$

Seven covariates of interest were assessed in subgroup analyses for their interaction with the effect of PORT on OS. A Bonferroni correction was applied, with a $P$ value of $.007(0.05 / 7)$ considered significant .

All statistical analyses were performed with $\mathrm{R}$ (version 3.4.0) with 2-sided tests and a significance level of .05.

\section{Results}

The baseline characteristics for the patients included in the study are listed in Table I. Patients receiving PORT had generally more aggressive features and were more likely to have higher T-classification $(P<.001)$, higher $\mathrm{N}$-classification $(P<.001)$, high-grade tumors $(P<.001)$, positive surgical margins $(P<.001)$, and ENE $(P<.001)$. Patients receiving PORT were also more likely to be male $(P<.001)$, have somewhat lower comorbidity $(P=.007)$, and receive treatment at nonacademic centers $(P<.001)$. In the group receiving PORT, those receiving chemotherapy in addition to PORT had generally more aggressive features (Supplemental Table S1, available in the online version of the article), including high-grade histology $(81.7 \%$ vs $57.5 \%, P<.001)$, T3-T4 tumors $(68.5 \%$ vs $52.9 \%, P<.001)$, N2-N3 disease $(73.5 \%$ vs $33.2 \%, P<.001)$, ENE $(55.6 \%$ vs $26.2 \%, P<.001)$, and positive margins $(48.4 \%$ vs $33.3 \%, P<.001)$.

The median follow-up time was 54 months. PORT was associated with significantly improved OS after adjusting for imbalances in other covariates in multivariable analysis (Table 2), although no benefit was seen with the addition of adjuvant chemotherapy (HR $=1.154 ; 95 \% \mathrm{CI}, 0.994-$ $1.339 ; P<.001)$. Because the effect of PORT varied over time and violated the Cox proportion hazards assumption, the effect of this variable was dichotomized into its effect within the first 9 months after diagnosis $(\mathrm{HR}=0.261 ; 95 \%$ CI, 0.201-0.338; $P<.001)$ and the effect $>9$ months after diagnosis $(\mathrm{HR}=0.753 ; 95 \% \mathrm{CI}, 0.657-0.863 ; P<.001)$ in the multivariable Cox model, with significant benefit seen in both intervals.

In propensity score-matched cohorts, 5-year OS was $67.1 \%$ (95\% CI, 64.0-70.4) with PORT and 60.6\% (95\% CI, 57.5-63.8) with surgery alone $(P<.001$; Figure $\mathbf{I})$.

In subgroup analyses, PORT was associated with significantly longer survival in virtually all subgroups of patients after multivariable adjustment (Figure 2).

To mitigate any potential contribution of immortal time bias, several sensitivity analyses were performed. In a landmark analysis of patients surviving at least 6 months after diagnosis (Supplemental Table S2, available in the online version of the article), PORT remained strongly associated with improved survival in multivariable analyses $(\mathrm{HR}=$ $0.741 ; 95 \%$ CI, $0.653-0.841 ; P<.001)$. Of note, the Cox proportional hazards assumption was no longer violated for PORT with this cohort. In propensity score-matched cohorts, 5-year OS was $70.5 \%$ and $60.5 \%$ among patients with and without PORT, respectively (Supplemental Figure $\mathrm{S} 1$, available in the online version of the article). Similar results were also observed when PORT was defined as any radiation after surgery, irrespective of dose delivered, both in the overall cohort (Supplemental Table S3, available in the online version of the article) and in the landmark cohort surviving at least 6 months (Supplemental Table S4 and Figure S2, available in the online version of the article).

\section{Discussion}

In the present study, we show that PORT is associated with a $6.5 \%$ increase in absolute 5 -year OS in propensity scorematched cohorts of patients with SGC who are undergoing surgical resection and neck dissection. Notably, we found that the magnitude of the survival effect associated with PORT varied over time, requiring the use of time-varying coefficients in our Cox regression model. The association of PORT with longer survival was strongest within the first 9 months after diagnosis, with PORT associated with a relative mortality decrease of approximately $75 \%$ in comparison with surgery alone. PORT continued to be associated with improved survival beyond 9 months from diagnosis but with a smaller relative decrease in mortality of $25 \%$. This variability over time is possibly related to the natural history of SGC, which includes multiple histologies that have a propensity for indolent distant metastasis and late relapse, ${ }^{19}$ potentially abrogating the survival impact of PORT at later time points. We further confirmed that PORT was associated with longer survival in landmark analyses of patients surviving at least 6 months after diagnosis to mitigate the effect of immortal time bias. Our study provides one of the largest and most statistically rigorous analyses supporting the benefit of PORT in SGC to date.

The association of PORT with longer survival was seen across essentially every subgroup in our study. Despite the broad benefit from PORT for essentially all patients in our study, it is important to note that we restricted our analysis to patients undergoing neck dissections removing at least 10 LNs. Therefore, virtually all patients likely harbored $\geq 1$ 
Table I. Baseline Characteristics of Patients with Salivary Gland Malignancies Undergoing Surgery with or without Postoperative Radiation Therapy.

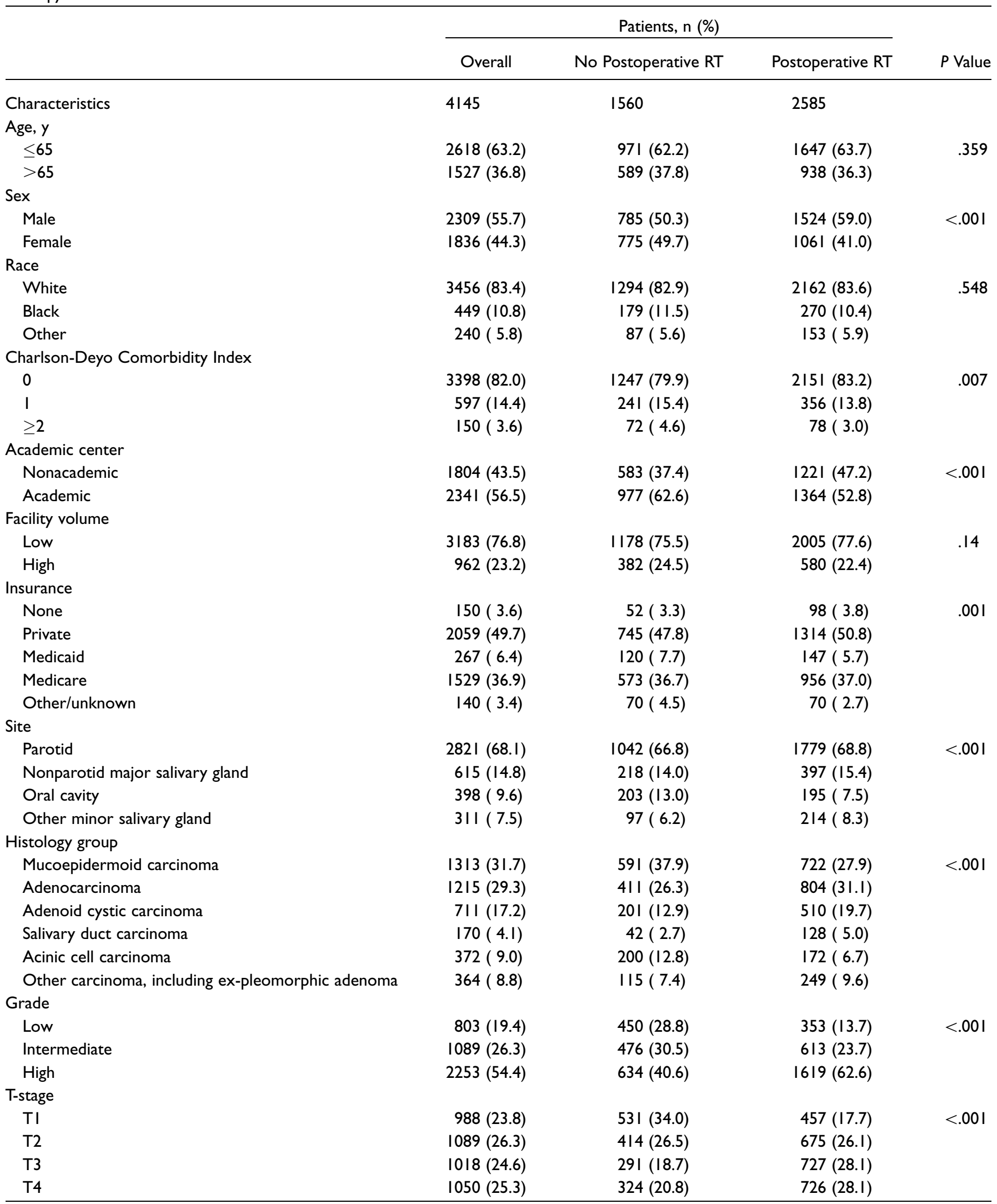


Table I. (continued)

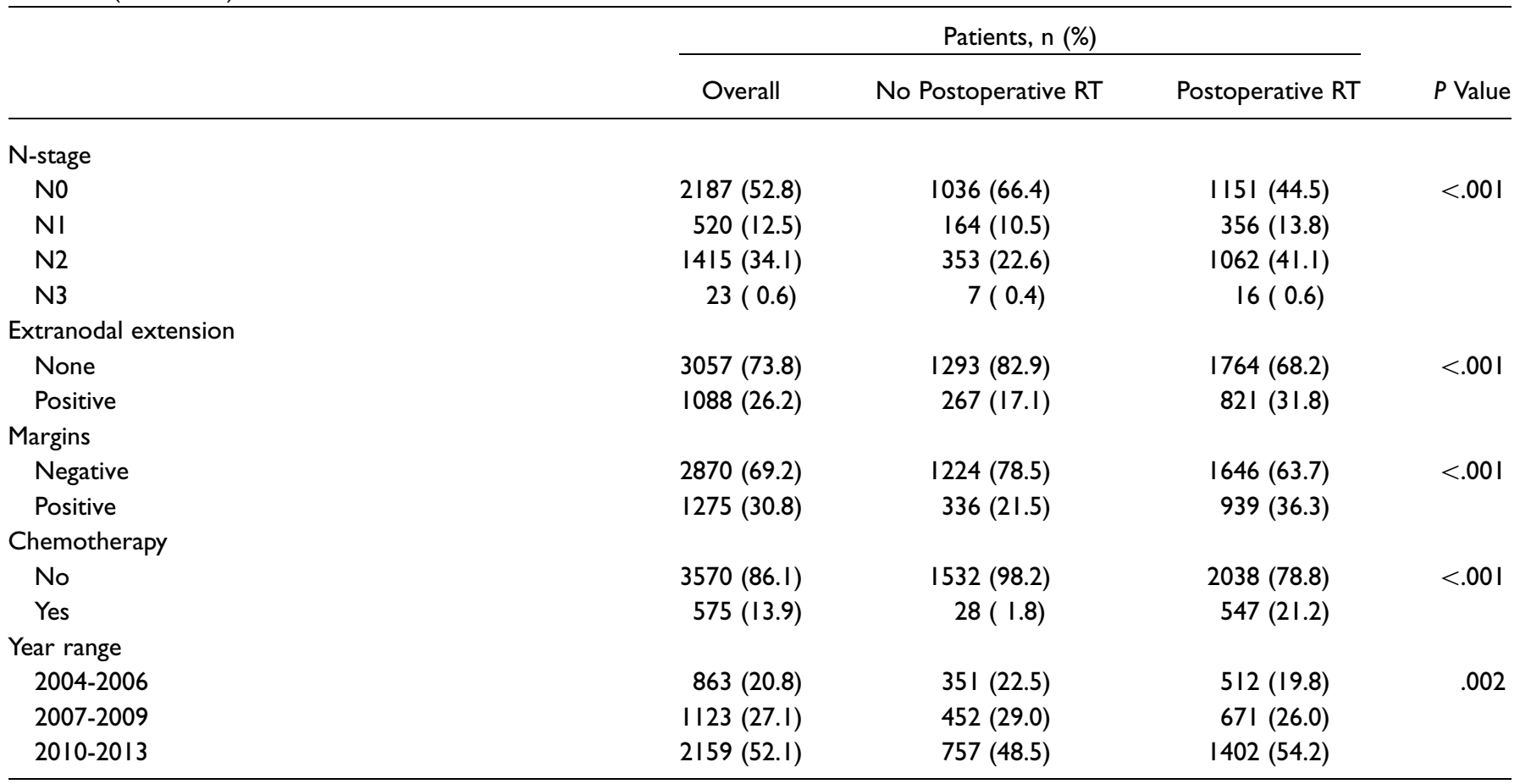

high-risk features that prompted more extensive surgery. Although our results strongly support PORT for all patients with high-risk features, they cannot be extrapolated to patients with lower-risk features who would not typically be recommended to undergo neck dissection.

SGCs are generally considered radioresistant. However, this notion comes predominantly from the disappointing local control observed with definitive radiation for unresectable tumors. ${ }^{20}$ In contrast, our study adds to a growing body of retrospective literature supporting radiation as a highly efficacious treatment for salivary tumors in the setting of microscopic disease. For example, in a study from the Netherlands, PORT dramatically increased 10-year local control in SGC by an absolute rate of $30 \%$ to $40 \%$ in subgroups of patients with adverse pathologic features, despite the fact that patients receiving surgery alone had generally more favorable characteristics. ${ }^{6}$ Similarly profound improvements in local control with PORT in SGC were reported by other studies. ${ }^{21-23}$ In addition to controlling microscopic local disease, radiation seems to be very effective at controlling microscopic regional disease as well. For example, in a retrospective study from the University of California, San Francisco, patients with salivary cancers undergoing surgical resection without neck dissection and PORT had $100 \%$ regional control at 10 years if elective neck irradiation was administered, as compared with $74 \%$ without elective neck irradiation. ${ }^{26}$ Although several studies observed a survival benefit with PORT in subsets of patients, ${ }^{21,25}$ it was not consistent. This discrepancy may be related to the small size of most series, given the rarity of this disease and the fact that patients undergoing surgery with radiation typically have more aggressive features as compared with patients treated with surgery alone.

Our study showed no evidence of prolonged survival associated with the addition of adjuvant chemotherapy. This is similar to what was reported in several other retrospective studies. $^{26-28}$ However, because patients with SGC who are undergoing adjuvant CRT typically have more high-risk features than those undergoing adjuvant radiation alone, it is difficult to draw definitive conclusions, and the role of chemotherapy remains controversial. The benefit of concomitant CRT in head and neck squamous cell carcinoma is one of the most well-established concepts in all of oncology, ${ }^{29-31}$ and it is possible that chemotherapy could radiosensitize SGC and provide at least a local control benefit. An ongoing phase II/III trial, RTOG 1008, is testing this hypothesis by randomizing patients with high-risk SGC after surgery to radiation with or without weekly cisplatin. ${ }^{32}$ Other systemic therapies of molecularly targeted agents, such as androgen deprivation therapy for androgen-receptor positive tumors $^{33}$ and Herceptin (trastuzumab) for HER2amplified tumors, ${ }^{34}$ may be promising agents to combine with PORT in SGC, although this is challenging to test given that it requires clinical trials involving a subset of an already rare disease. Nevertheless, because the survival of patients with SGC has not improved greatly over the past several decades, developing systemic therapeutic strategies to optimize locoregional control and eliminate micrometastatic disease is a critical unmet need in SGC. ${ }^{35}$

There are multiple limitations of this study. First, this is a retrospective observational study. Selection bias may 
Table 2. Univariate and Multivariable Predictors of Outcome for Overall Survival among Patients with Salivary Gland Malignancies Undergoing Surgery and Neck Dissection.

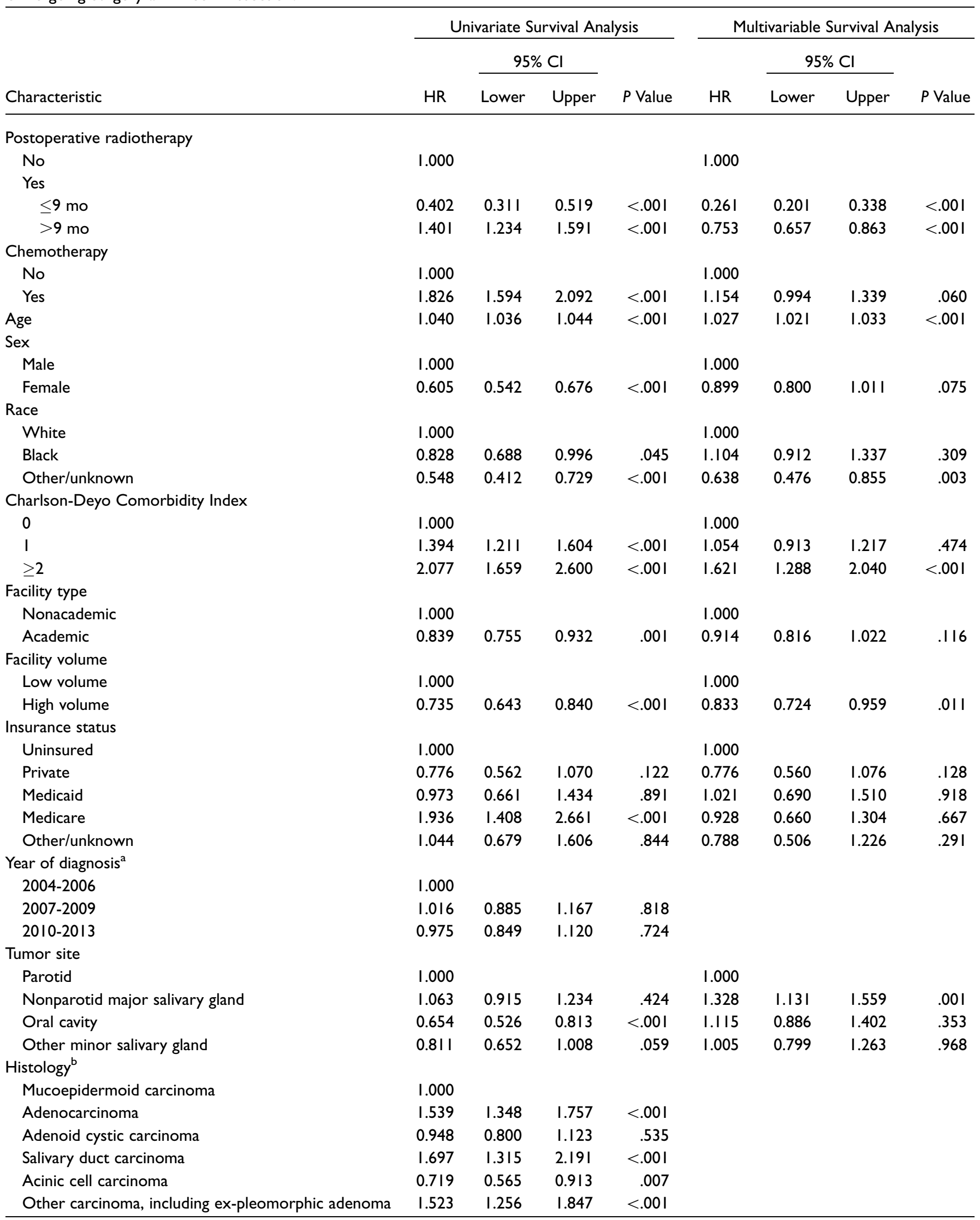


Table 2. (continued)

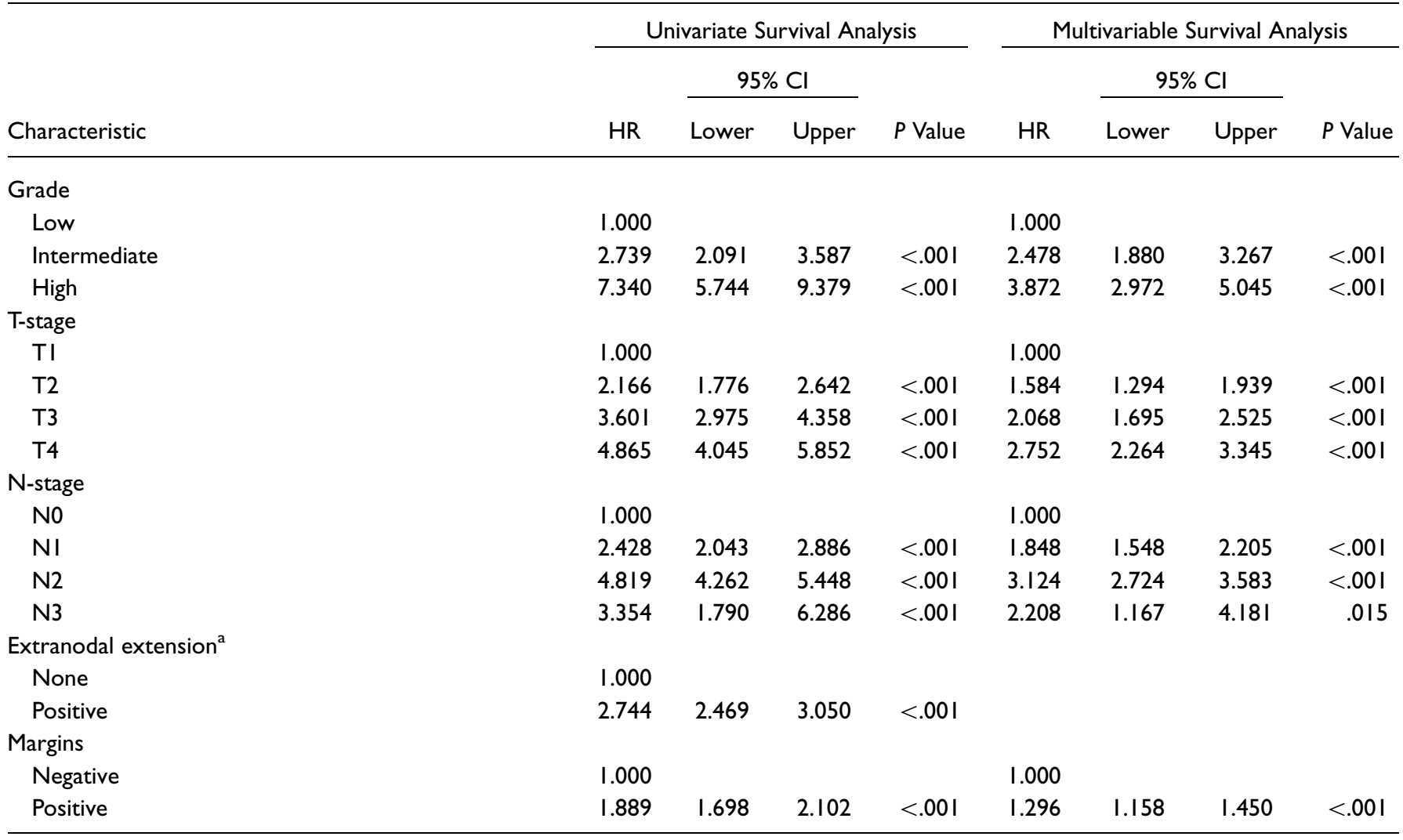

Abbreviation: $\mathrm{HR}$, hazard ratio.

${ }^{\text {a } D r o p p e d ~ f r o m ~ m o d e l . ~}$

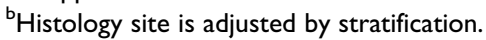

A

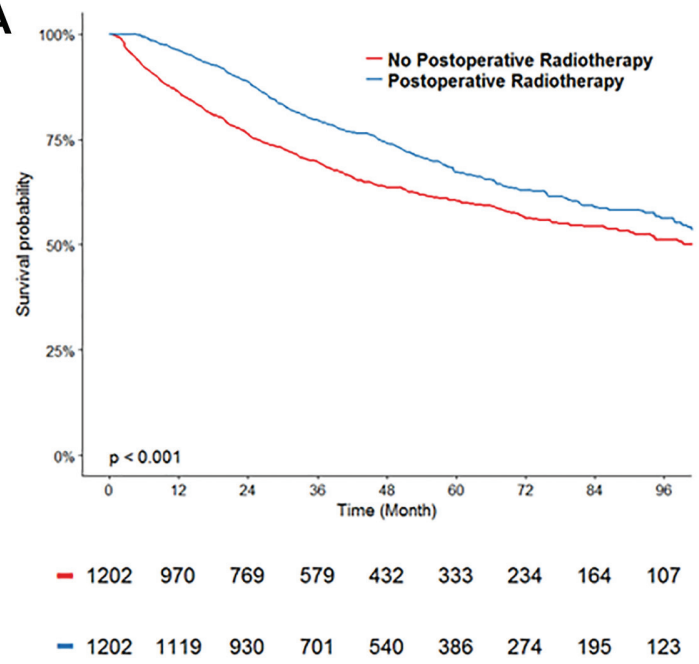

B

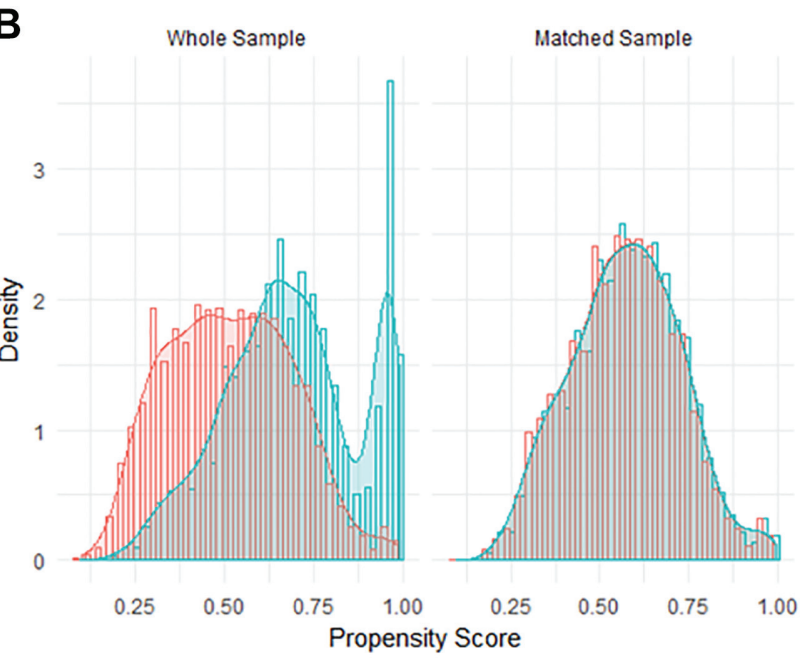

Figure I. (A) Overall survival of patients with salivary gland carcinoma, with or without postoperative radiation therapy, in propensity score-matched cohorts. (B) Histogram of propensity scores before and after matching.

exist, and the decisions to administer RT or decline it are not always obvious. However, it is notable that virtually every clinical and pathologic factor favored patients receiving observation after surgery in comparison with PORT, making it less likely that unmeasured confounding variables - including known prognostic factors not captured in the NCDB (eg, perineural invasion) - are driving the results that we observed. Also, SGC is highly heterogeneous in terms of histology, grade, and anatomic site. We adjusted for these factors in all analyses, but unmeasured 


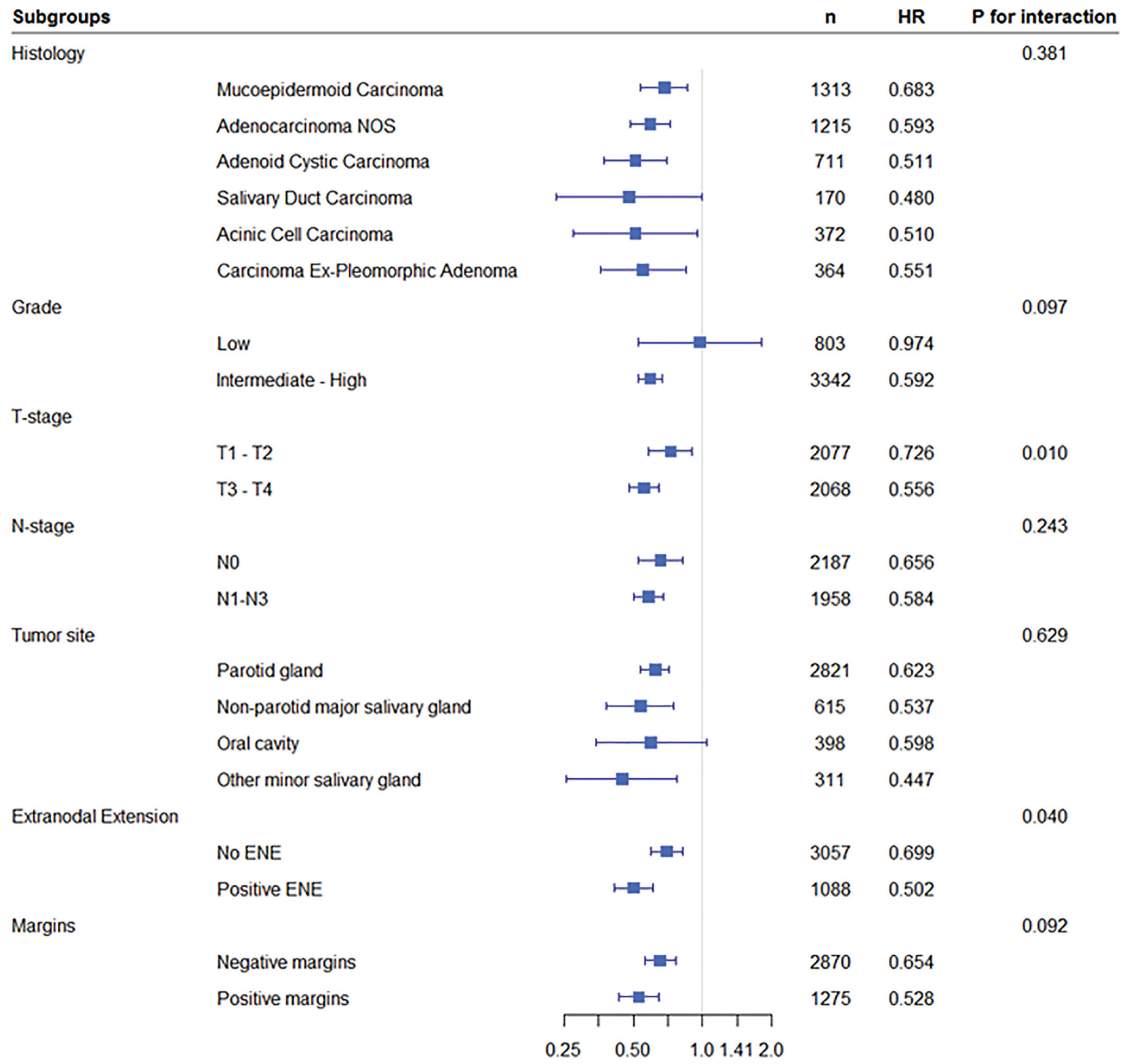

Figure 2. Multivariate Cox regression of the association of postoperative radiation with overall survival for patients with salivary gland carcinoma undergoing surgical resection and neck dissection in subgroups of interest. $P$ values represent tests of interaction. Error bars indicate $95 \% \mathrm{Cl}$. ENE, extranodal extension; HR, hazard ratio; NOS, not otherwise specified.

heterogeneity could influence that benefit of PORT. Next, we note that our results are not applicable to the large proportion of indolent salivary tumors that do not require neck dissection. Several important variables were not available for all patients, including grade, ENE, and margins. We accounted for these missing values by performing multiple imputation. We excluded patients who did not undergo neck dissections to ensure complete pathologic staging and allow for more accurate matching between patients who did and did not receive PORT. However, this limits the applicability of our results to low-risk salivary cancers resected without neck dissection. NCDB also lacks cancer-specific outcomes, such as local control, regional control, and distant metastasis. Despite these limitations, we believe that our observation of improved survival associated with PORT in SGC with surgical resection and neck dissection is robust.

\section{Conclusions}

PORT is associated with improved survival among patients with SGC who are undergoing surgical resection and neck dissection. Longer survival with PORT was seen for virtually all patients in our study, and when using landmark analysis to mitigate the effect of immortal time bias. Importantly, these results are not applicable to low-risk SGCs that do not require neck dissection. Notably, adjuvant chemotherapy had no association with improved survival. Future studies attempting to develop effective systemic therapy regimens for sterilization of micrometastasis are needed 
to further improve outcomes in this heterogeneous group of diseases.

\section{Author Contributions}

Katri Aro, conception/design, data analysis/interpretation, drafting/revision manuscript, final manuscript approval, agrees to be accountable; Allen S. Ho, data interpretation, revising manuscript, final manuscript approval, agrees to be accountable; Michael Luu, data analysis, drafting/revision manuscript, final manuscript approval, agrees to be accountable; Sungjin Kim, data interpretation, revising manuscript, final manuscript approval, agrees to be accountable; Mourad Tighiouart, data interpretation, revising manuscript, final manuscript approval, agrees to be accountable; Emi J. Yoshida, data interpretation, revising manuscript, final manuscript approval, agrees to be accountable; Jon Mallen-St Clair, data interpretation, revising manuscript, final manuscript approval, agrees to be accountable; Stephen L. Shiao, data interpretation, revising manuscript, final manuscript approval, agrees to be accountable; Ilmo Leivo, data interpretation, revising manuscript, final manuscript approval, agrees to be accountable; Zachary S. Zumsteg, conception/design, data analysis/interpretation, drafting/revision manuscript, final manuscript approval, agrees to be accountable.

\section{Disclosures}

Competing interests: Zachary S. Zumsteg, EMD Seronoconsultant; Scripps Proton Therapy Centerexternal — advisory board.

Sponsorships: None.

Funding source: Katri Aro has been supported by the Sigrid Jusélius Foundation and the Finnish Otorhinolaryngology Research Foundation.

\section{Supplemental Material}

Additional supporting information is available in the online version of the article.

\section{References}

1. Barnes L, Eveson JW, Reichart P, Sidransky D, eds. World Health Organization classification of tumours. In: Tumours of the Salivary Glands. Lyon, France: IARC Press; 2005.

2. El-Naggar AK, Chan JKC, Gramdis JR, Takata T, Slootweg PJ, editor. World Health Organization classification of tumours. In: Tumours of the Salivary Glands. Lyon, France: IARC Press; 2017.

3. Garden AS, el-Naggar AK, Morrison WH, Callender DL, Ang KK, Peters LJ. Postoperative radiotherapy for malignant tumors of the parotid gland. Int J Radiat Oncol Biol Phys. 1997;37:79-85.

4. Mahmood U, Koshy M, Goloubeva O, Suntharalingam M. Adjuvant radiation therapy for high-grade and/or locally advanced major salivary gland tumors. Arch Otolaryngol Head Neck Surg. 2011;137:1025-1030.

5. Terhaard $\mathrm{CH}$. Postoperative and primary radiotherapy for salivary gland carcinomas: indications, techniques, and results. Int J Radiat Oncol Biol Phys. 2007;69:S52-S55.

6. Terhaard $\mathrm{CH}$, Lubsen $\mathrm{H}$, Rasch $\mathrm{CR}$, et al. The role of radiotherapy in the treatment of malignant salivary gland tumors. Int J Radiat Oncol Biol Phys. 2005;61:103-111.
7. Jegadeesh N, Liu Y, Prabhu RS, et al. Outcomes and prognostic factors in modern era management of major salivary gland cancer. Oral Oncol. 2015;51:770-777.

8. Safdieh J, Givi B, Osborn V, Lederman A, Schwartz D, Schreiber D. Impact of adjuvant radiotherapy for malignant salivary gland tumors. Otolaryngol Head Neck Surg. 2017; 157:988-994.

9. Cheraghlou S, Kuo P, Mehra S, et al. Adjuvant therapy in major salivary gland cancers: analysis of 8580 patients in the National Cancer Database. Head Neck. 2018;40:1343-1355.

10. Bakst RL, Su W, Ozbek U, et al. Adjuvant radiation for salivary gland malignancies is associated with improved survival: a National Cancer Database analysis. Adv Radiat Oncol. 2017; 2:159-166.

11. Steele GD Jr, Osteen RT, Winchester DP, Murphy GP, Menck HR. Clinical highlights from the National Cancer Data Base: 1994. CA Cancer J Clin. 1994;44:71-80.

12. Little R. A test of missing completely at random for multivariable data with missing values. Journal of the American Statistical Association. 1988;83:1198-1202.

13. van Buuren S, Groothuis-Oudshoorn K. Mice: multivariate imputation by chained equations in R. Journal of Statistical Software. 2011;45:1-67.

14. van Buuren S. Multiple imputation of discrete and continuous data by fully conditional specification. Statistical Methods in Medical Research. 2007;16:219-242.

15. Yamashita T, Yamashita K, Kamimura R. A stepwise AIC method for variable selection in linear regression. Communications in Statistics: Theory and Methods. 2007;36: 2395-2403.

16. Hess KR. Graphical methods for assessing violations of the proportional hazards assumption in Cox regression. Stat Med. 1995;14:1707-1723.

17. Kleinbaum DG. Survival analysis, a self-learning text. Biometrical Journal. 1998;40:107-108.

18. Austin PC. Optimal caliper widths for propensity-score matching when estimating differences in means and differences in proportions in observational studies. Pharm Stat. 2011;10:150-161.

19. Chen AM, Garcia J, Granchi PJ, Johnson J, Eisele DW. Late recurrence from salivary gland cancer: when does "cure" mean cure? Cancer. 2008;112:340-344.

20. Laramore GE, Krall JM, Griffin TW, et al; Radiation Therapy Oncology Group, Medical Research Council. Neutron versus photon irradiation for unresectable salivary gland tumors: final report of an RTOG-MRC randomized clinical trial. Int $J$ Radiat Oncol Biol Phys. 1993;27:235-240.

21. Armstrong JG, Harrison LB, Spiro RH, Fass DE, Strong EW, Fuks ZY. Malignant tumors of major salivary gland origin: a matched-pair analysis of the role of combined surgery and postoperative radiotherapy. Arch Otolaryngol Head Neck Surg. 1990;116:290-293.

22. Cho JK, Lim BW, Kim EH, et al. Low-grade salivary gland cancers: treatment outcomes, extent of surgery and indications for postoperative adjuvant radiation therapy. Ann Surg Oncol. 2016;23:4368-4375.

23. Chen AM, Garcia J, Bucci MK, Quivey JM, Eisele DW. The role of postoperative radiation therapy in carcinoma ex 
pleomorphic adenoma of the parotid gland. Int J Radiat Oncol Biol Phys. 2007;67:138-143.

24. Chen AM, Garcia J, Lee NY, Bucci MK, Eisele DW. Patterns of nodal relapse after surgery and postoperative radiation therapy for carcinomas of the major and minor salivary glands: what is the role of elective neck irradiation? Int $J$ Radiat Oncol Biol Phys. 2007;67:988-994.

25. Zeidan YH, Pekelis L, An Y, et al. Survival benefit for adjuvant radiation therapy in minor salivary gland cancers. Oral Oncol. 2015;51:438-445.

26. Amini A, Waxweiler TV, Brower JV, et al. Association of adjuvant chemoradiotherapy vs radiotherapy alone with survival in patients with resected major salivary gland carcinoma: data From the National Cancer Data Base. JAMA Otolaryngol Head Neck Surg. 2016;142:1100-1110.

27. Gebhardt BJ, Ohr JP, Ferris RL, et al. Concurrent chemoradiotherapy in the adjuvant treatment of high-risk primary salivary gland malignancies. Am J Clin Oncol. 2018;41:888-893.

28. Tanvetyanon T, Fisher K, Caudell J, Otto K, Padhya T, Trotti A. Adjuvant chemoradiotherapy versus with radiotherapy alone for locally advanced salivary gland carcinoma among older patients. Head Neck. 2016;38:863-870.

29. Bernier J, Domenge C, Ozsahin M, et al. Postoperative irradiation with or without concomitant chemotherapy for locally advanced head and neck cancer. N Engl J Med. 2004;350:1945-1952.
30. Pignon JP, Bourhis J, Domenge C, Designe L; MACH-NC Collaborative Group. Chemotherapy added to locoregional treatment for head and neck squamous-cell carcinoma: three meta-analyses of updated individual data. Meta-analysis of chemotherapy on head and neck cancer. Lancet. 2000;355: 949-955.

31. Zumsteg ZS, Kim S, David JM, et al. Impact of concomitant chemoradiation on survival for patients with T1-2N1 head and neck cancer. Cancer. 2017;123:1555-1565.

32. Radiation Therapy Oncology Group. RTOG 1008 protocol information. http://adcl.mdanderson.org/RPC/credentialing/files/ 1008.pdf. Accessed November 16, 2018.

33. Dalin MG, Watson PA, Ho AL, Morris LG. Androgen receptor signaling in salivary gland cancer. Cancers (Basel). 2017;9(2): E17.

34. Wang K, Russell JS, McDermott JD, et al. Profiling of 149 salivary duct carcinomas, carcinoma ex pleomorphic adenomas, and adenocarcinomas, not otherwise specified reveals actionable genomic alterations. Clin Cancer Res. 2016;22: 6061-6068.

35. Jensen AD, Nikoghosyan AV, Lossner K, et al. COSMIC: a regimen of intensity modulated radiation therapy plus doseescalated, raster-scanned carbon ion boost for malignant salivary gland tumors - results of the prospective phase 2 trial. Int J Radiat Oncol Biol Phys. 2015;93:37-46. 